\title{
A Representative Solution to $m$-Order Linear Ordinary Differential Equation with Nonlocal Conditions by Green's Functional Concept
}

\author{
Kemal Özen ${ }^{a, b}$ and Kamil Oruçoğlu ${ }^{a}$ \\ ${ }^{a} \dot{I}$ stanbul Technical University \\ Department of Mathematics, 34469 Maslak, İstanbul, Turkey \\ ${ }^{b}$ Namık Kemal University \\ Department of Mathematics, 59030 Tekirdağ, Turkey \\ E-mail(corresp.): ozenke@itu.edu.tr \\ E-mail: koruc@itu.edu.tr
}

Received February 11, 2012; revised June 29, 2012; published online September 1, 2012

\begin{abstract}
In this work, we investigate a linear completely nonhomogeneous nonlocal multipoint problem for an $m$-order ordinary differential equation with generally variable nonsmooth coefficients satisfying some general properties such as $p$-integrability and boundedness. A system of $m+1$ integro-algebraic equations called the special adjoint system is constructed for this problem. Green's functional is a solution of this special adjoint system. Its first component corresponds to Green's function for the problem. The other components correspond to the unit effects of the conditions. A solution to the problem is an integral representation which is based on using this new Green's functional. Some illustrative implementations and comparisons are provided with some known results in order to demonstrate the advantages of the proposed approach.
\end{abstract}

Keywords: Green's function, nonlocal boundary condition, nonlocal integral condition, boundary value problem, adjoint problem.

AMS Subject Classification: 34B05; 34B10; 34B27.

\section{Introduction}

Linear nonhomogeneous boundary value problems for an ordinary differential equation with generally variable coefficients involving nonlocal multipoint conditions of derivative or integral type arise in applied mathematics, physics, engineering and the various areas of mechanics such as theories of elasticity, elastic stability, plates and shells [28]. For example, the vibrations of wires and bridges composed of many parts of different densities can mathematically be modeled by such problems. Therefore, some demands and requirements for the 
construction of a solution to these problems in such areas make linear operator theory and generalized functions' theory valuable in mathematical analysis. Green's solution for such problems is an analytical solution which is principally based on these theories of mathematical analysis [14, 16, 24, 25].

The existence and uniqueness of solutions to nonlocal multipoint problems for an $m$-order ordinary differential equation have been studied in $[9,10,15]$. Green's function is a helpful tool for determining the existence and uniqueness of solutions to boundary value problems. Its construction for problems involving nonclassical conditions is an area which is recently improved. The problems in $[4,17,18,20,21,22,23,27]$ can be considered as relevant and valuable examples in this area. On the other hand, in [26], a second-order Sturm-Liouville problem with two nonlocal boundary conditions has been investigated and the condition for existence Green's function in the space of parameters has been obtained.

Some classical methods exist in literature to construct Green's function. However, these methods can cause serious difficulties in a problem, concentrated by this work, in case of existence of nonsmooth coefficients and nonclassical conditions, or in case of nonexistence of formal adjoint operator for the equation's operator, or in case of existence of any extension for the equation's operator only on a space of distributions. In order to minimize these difficulties, in this work, a representative solution to a linear completely nonhomogeneous nonlocal multipoint problem for an $m$-order ordinary differential equation with generally variable nonsmooth coefficients satisfying some general properties such as $p$-integrability and boundedness is constructed by using Green's functional approach [5]. This approach is based on [1, 2, 6] and some methods of functional analysis, and it is principally different from the classical methods [25]. A special adjoint system corresponding to the problem is constructed in accordance with this approach. The structure of this system depends on the operators not on the homogeneity in the problem. Fredholm alternative is utilized for derivation of solvability conditions of the problem.

Another useful analytical method which minimizes these difficulties on the construction of Green's function is based on variation of parameters for ordinary differential equations [29]. Green's functions for some nonlocal problems have been obtained by using this method in [20,21, 22, 27]. But, an investigation extended to an $m$-order ordinary differential equation in these four papers does not exist except for [20] in which an extension to the problem involving nonlocal and local conditions of two Samarskii-Bitsadze-type and $m-2$ classical-type has been presented. Therefore, in our work, we principally aim to extend the approach in $[5,6]$ to an $m$-order linear ordinary differential equation with complicated nonlocal boundary conditions in order to construct its Green's solution.

The rest of our work is organized as follows: In Section 2, the main problem considered throughout the work is stated in detail. In Sections 3 and 4, the adjoint space of the solution space and the adjoint system of integro-algebraic equations are introduced respectively. In Section 5, the solvability conditions for the completely nonhomogeneous problem are concentrated. In Section 6, Green's functional and the special adjoint system are defined. In Section 7, 
some applications and comparisons are provided. In Section 8, the conclusions and some advantages are emphasized.

\section{Statement of the Problem}

Let $\mathbb{R}$ be the set of real numbers. Let $G=\left(x_{0}, x_{1}\right)$ be a bounded open interval in $\mathbb{R}$. Let $L_{p}(G)$ with $1 \leq p<\infty$ be the space of $p$-integrable functions on $G$. Let $L_{\infty}(G)$ be the space of measurable and essentially bounded functions on $G$, and let $W_{p}^{(m)}(G)$ with $1 \leq p \leq \infty$ be the space of all functions $u=u(x) \in$ $L_{p}(G)$ having derivatives $d^{k} u / d x^{k} \in L_{p}(G)$, where $k=1,2, \ldots, m$. The norm on the space $W_{p}^{(m)}(G)$ is defined as

$$
\|u\|_{W_{p}^{(m)}(G)}=\sum_{k=0}^{m}\left\|\frac{d^{k} u}{d x^{k}}\right\|_{L_{p}(G)} .
$$

The other structural properties of the space $W_{p}=W_{p}^{(m)}(G)$ are given in the next sections. We consider the nonlocal multipoint boundary value problem which can be transformed into the $m$-order linear ordinary differential equation

$$
\left(V_{m} u\right)(x) \equiv u^{(m)}(x)+\sum_{i=0}^{m-1} A_{i}(x) u^{(i)}(x)=z_{m}(x), \quad x \in G,
$$

subject to the following generally nonlocal boundary conditions

$$
\begin{aligned}
V_{m-1} u \equiv & \sum_{i=0}^{m-1} a_{m-1}^{i} u^{(i)}\left(x_{0}\right)+\int_{x_{0}}^{x_{1}} g_{m-1}(\xi) u^{(m)}(\xi) d \xi=z_{m-1}, \\
V_{m-2} u \equiv & \sum_{i=0}^{m-1} a_{m-2}^{i} u^{(i)}\left(x_{0}\right)+\int_{x_{0}}^{x_{1}} g_{m-2}(\xi) u^{(m)}(\xi) d \xi=z_{m-2}, \\
& \ldots \\
V_{1} u \equiv & \sum_{i=0}^{m-1} a_{1}^{i} u^{(i)}\left(x_{0}\right)+\int_{x_{0}}^{x_{1}} g_{1}(\xi) u^{(m)}(\xi) d \xi=z_{1}, \\
V_{0} u \equiv & \sum_{i=0}^{m-1} a_{0}^{i} u^{(i)}\left(x_{0}\right)+\int_{x_{0}}^{x_{1}} g_{0}(\xi) u^{(m)}(\xi) d \xi=z_{0},
\end{aligned}
$$

where $u^{(j)}$ denotes the derivative of order $j$ for $u$ with respect to its variable and $u^{(0)}=u$. We investigate a solution, which is given as an integral representation, to problem $(2.1)-(2.2)$ in the space $W_{p}$. Furthermore, we assume that the following conditions are satisfied: $A_{i} \in L_{p}(G)$ and $g_{i} \in L_{q}(G)$ for $i=0,1, \ldots, m-1$ are given functions; $a_{m-1}^{i}, a_{m-2}^{i}, \ldots, a_{1}^{i}, a_{0}^{i}$ for $i=$ $0,1, \ldots, m-1$ are given real numbers; $z_{m} \in L_{p}(G)$ is a given function and $z_{i}$ for $i=0,1, \ldots, m-1$ are given real numbers.

Problem (2.1)-(2.2) is a linear completely nonhomogeneous problem which can be considered as an operator equation:

$$
V u=z,
$$


with the linear operator $V=\left(V_{m}, V_{m-1}, \ldots, V_{0}\right)$ and $z=\left(z_{m}(x), z_{m-1}, \ldots, z_{0}\right)$.

The conditions given above guarantee that $V$ is bounded from $W_{p}$ into the Banach space

$$
E_{p} \equiv L_{p}(G) \times \underbrace{\mathbb{R} \times \cdots \times \mathbb{R}}_{m \text { times }}
$$

of $(m+1)$-tuples $z=\left(z_{m}(x), z_{m-1}, \ldots, z_{0}\right)$ with

$$
\|z\|_{E_{p}}=\left\|z_{m}\right\|_{L_{p}(G)}+\left|z_{m-1}\right|+\cdots+\left|z_{0}\right|, \quad 1 \leq p \leq \infty .
$$

If, for a given $z \in E_{p}$, the problem (2.1)-(2.2) has a unique solution $u \in W_{p}$ with $\|u\|_{W_{p}} \leq c_{0}\|z\|_{E_{p}}$, then this problem is called a well-posed problem, where $c_{0}$ is a constant independent of $z$. Problem (2.1)-(2.2) is well-posed if and only if $V: W_{p} \rightarrow E_{p}$ is a (linear) homeomorphism.

\section{Adjoint Space of the Solution Space}

Problem (2.1)-(2.2) is investigated by means of a new concept of the adjoint problem. This concept is introduced in the papers $[2,6]$ by the adjoint operator $V^{*}$ of $V$. Furthermore, some isomorphic decompositions of the space $W_{p}$ of solutions and its adjoint space $W_{p}^{*}$ are employed. Any function $u \in W_{p}$ can be represented as

$$
u(x)=\sum_{i=0}^{m-1} u^{(i)}(\alpha) \frac{(x-\alpha)^{i}}{i !}+\int_{\alpha}^{x} \frac{(x-\xi)^{m-1}}{(m-1) !} u^{(m)}(\xi) d \xi,
$$

where $\alpha$ is a given point in $\bar{G}$ which is the set of closure points for $G$. Furthermore, the trace or value operators $D_{0} u=u(\gamma), D_{1} u=u^{\prime}(\gamma), \ldots, D_{m-1} u=$ $u^{(m-1)}(\gamma)$ are bounded and surjective from $W_{p}$ onto $\mathbb{R}$ for a given point $\gamma$ of $\bar{G}$. In addition, the values $u(\alpha), u^{\prime}(\alpha), \ldots, u^{(m-1)}(\alpha)$ and the derivative $u^{(m)}(x)$ are unrelated elements of the function $u \in W_{p}$ in the following sense: for any real numbers $\nu_{0}, \nu_{1}, \ldots, \nu_{m-1}$ and any function $\nu_{m} \in L_{p}(G)$, there exists one and only one $u \in W_{p}$ such that $u(\alpha)=\nu_{0}, u^{\prime}(\alpha)=\nu_{1}, \ldots, u^{(m-1)}(\alpha)=\nu_{m-1}$ and $u^{(m)}(x)=\nu_{m}(x)$. Therefore, there exists a linear homeomorphism between $W_{p}$ and $E_{p}$. In other words, the space $W_{p}$ has the isomorphic decomposition

$$
W_{p}=L_{p}(G) \times \underbrace{\mathbb{R} \times \cdots \times \mathbb{R}}_{m \text { times }} .
$$

Theorem 1. If $1 \leq p<\infty$, then any linear bounded functional $F \in W_{p}^{*}$ can be represented as

$$
F(u)=\int_{x_{0}}^{x_{1}} u^{(m)}(x) \varphi_{m}(x) d x+\sum_{i=0}^{m-1} u^{(i)}\left(x_{0}\right) \varphi_{i}
$$

with a unique element $\varphi=\left(\varphi_{m}(x), \varphi_{m-1}, \varphi_{m-2}, \ldots, \varphi_{0}\right) \in E_{q}$ where $p+q=p q$. Any linear bounded functional $F \in W_{\infty}^{*}$ can be represented as

$$
F(u)=\int_{x_{0}}^{x_{1}} u^{(m)}(x) d \varphi_{m}+\sum_{i=0}^{m-1} u^{(i)}\left(x_{0}\right) \varphi_{i}
$$


with a unique element $\varphi=\left(\varphi_{m}(e), \varphi_{m-1}, \varphi_{m-2}, \ldots, \varphi_{0}\right) \in \widehat{E_{1}}=\left(B A\left(\sum, \mu\right)\right) \times$ $\mathbb{R} \times \cdots \times \mathbb{R}$ where $\mu$ is the Lebesgue measure on $\mathbb{R}, \sum$ is $\sigma$-algebra of the $\mu$ measurable subsets $e \subset G$ and $B A\left(\sum, \mu\right)$ is the space of all bounded additive functions $\varphi_{m}(e)$ defined on $\sum$ with $\varphi_{m}(e)=0$ when $\mu(e)=0$ [12]. The inverse is also valid, that is, if $\varphi \in E_{q}$, then (3.2) is bounded on $W_{p}$ for $1 \leq p<\infty$ and $p+q=p q$. If $\varphi \in \widehat{E_{1}}$, then (3.3) is bounded on $W_{\infty}$.

Proof. The operator $N u \equiv\left(u^{(m)}(x), u^{(m-1)}\left(x_{0}\right), \ldots, u\left(x_{0}\right)\right): W_{p} \rightarrow E_{p}$ is bounded and has a bounded inverse $N^{-1}$ represented by

$$
\begin{aligned}
u(x) & =\left(N^{-1} h\right)(x) \equiv \int_{x_{0}}^{x} \frac{(x-\xi)^{m-1}}{(m-1) !} h_{m}(\xi) d \xi+\sum_{i=0}^{m-1} h_{i} \frac{\left(x-x_{0}\right)^{i}}{i !} \\
h & =\left(h_{m}(x), h_{m-1}, \ldots, h_{0}\right) \in E_{p}
\end{aligned}
$$

The kernel Ker $N$ of $N$ is trivial and the image $\operatorname{Im} N$ of $N$ is equal to $E_{p}$. Hence, there exists a bounded adjoint operator $N^{*}: E_{p}^{*} \rightarrow W_{p}^{*}$ with $\operatorname{Ker} N^{*}=$ $\{0\}$ and $\operatorname{Im} N^{*}=W_{p}^{*}$. In other words, for a given $F \in W_{p}^{*}$ there exists a unique $\psi \in E_{p}^{*}$ such that

$$
F=N^{*} \psi \quad \text { or } \quad F(u)=\psi(N u), \quad u \in W_{p}
$$

If $1 \leq p<\infty$, then $E_{p}^{*}=E_{q}$ in the sense of an isomorphism [12]. Therefore, the functional $\psi$ can be represented by

$$
\psi(h)=\int_{x_{0}}^{x_{1}} \varphi_{m}(x) h_{m}(x) d x+\sum_{i=0}^{m-1} \varphi_{i} h_{i}, \quad h \in E_{p},
$$

with a unique element $\varphi=\left(\varphi_{m}(x), \varphi_{m-1}, \ldots, \varphi_{0}\right) \in E_{q}$. By expressions (3.5) and (3.6), any $F \in W_{p}^{*}$ can uniquely be represented by (3.2). For a given $\varphi \in E_{q}$, the functional $F$ represented by (3.2) is bounded on $W_{p}$. Hence, (3.2) is a general form for the functional $F \in W_{p}^{*}$.

The proof for the case $p=\infty$ can be shown similarly.

Theorem 1 guarantees that $W_{p}^{*}=E_{q}$ for all $1 \leq p<\infty$, and $W_{\infty}^{*}=$ $E_{\infty}^{*}=\widehat{E_{1}}$. The space $E_{1}$ can also be considered as a subspace of the space $\widehat{E_{1}}$.

\section{Adjoint System of Integro-Algebraic Equations}

Investigating an explicit form for the adjoint operator $V^{*}$ of $V$ is taken into consideration in this section. To this end, any $f=\left(f_{m}(x), f_{m-1}, \ldots, f_{0}\right) \in E_{q}$ is taken as a linear bounded functional on $E_{p}$ and also

$$
f(V u) \equiv \int_{x_{0}}^{x_{1}} f_{m}(x)\left(V_{m} u\right)(x) d x+\sum_{i=0}^{m-1} f_{i}\left(V_{i} u\right), \quad u \in W_{p}
$$


can be supposed. By substituting expressions (2.1) and (2.2), and expression (3.1) (for $\alpha=x_{0}$ ) of $u \in W_{p}$ into (4.1), we have

$$
\begin{aligned}
& f(V u) \equiv \int_{x_{0}}^{x_{1}} f_{m}(x)\left[u^{(m)}(x)+A_{m-1}(x)\left\{u^{(m-1)}\left(x_{0}\right)+\int_{x_{0}}^{x} u^{(m)}(\xi) d \xi\right\}\right. \\
& +A_{m-2}(x)\left\{u^{(m-2)}\left(x_{0}\right)+u^{(m-1)}\left(x_{0}\right)\left(x-x_{0}\right)+\int_{x_{0}}^{x}(x-\xi) u^{(m)}(\xi) d \xi\right\} \\
& +\cdots+A_{1}(x)\left\{\sum_{i=1}^{m-1} u^{(i)}\left(x_{0}\right) \frac{\left(x-x_{0}\right)^{i-1}}{(i-1) !}+\int_{x_{0}}^{x} \frac{(x-\xi)^{m-2}}{(m-2) !} u^{(m)}(\xi) d \xi\right\} \\
& \left.+A_{0}(x)\left\{\sum_{i=0}^{m-1} u^{(i)}\left(x_{0}\right) \frac{\left(x-x_{0}\right)^{i}}{i !}+\int_{x_{0}}^{x} \frac{(x-\xi)^{m-1}}{(m-1) !} u^{(m)}(\xi) d \xi\right\}\right] d x \\
& +\sum_{i=0}^{m-1} f_{i}\left\{\sum_{j=0}^{m-1} a_{i}^{j} u^{(j)}\left(x_{0}\right)+\int_{x_{0}}^{x_{1}} g_{i}(\xi) u^{(m)}(\xi) d \xi\right\} .
\end{aligned}
$$

After some arrangements, we can obtain

$$
\begin{aligned}
f(V u) & \equiv \int_{x_{0}}^{x_{1}} f_{m}(x)\left(V_{m} u\right)(x) d x+\sum_{i=0}^{m-1} f_{i}\left(V_{i} u\right) \\
& =\int_{x_{0}}^{x_{1}}\left(w_{m} f\right)(\xi) u^{(m)}(\xi) d \xi+\sum_{i=0}^{m-1}\left(w_{i} f\right) u^{(i)}\left(x_{0}\right) \\
& \equiv(w f)(u), \quad \forall f \in E_{q}, \quad \forall u \in W_{p}, \quad 1 \leq p \leq \infty,
\end{aligned}
$$

where

$$
\begin{aligned}
& \left(w_{m} f\right)(\xi)=f_{m}(\xi)+\sum_{j=0}^{m-1} f_{j} g_{j}(\xi)+\int_{\xi}^{x_{1}} f_{m}(s)\left\{\sum_{i=0}^{m-1} A_{i}(s) \frac{(s-\xi)^{m-1-i}}{(m-1-i) !}\right\} d s, \\
& w_{l} f=\sum_{j=0}^{m-1} f_{j} a_{j}^{l}+\int_{x_{0}}^{x_{1}} f_{m}(s)\left\{\sum_{i=0}^{l} A_{i}(s) \frac{\left(s-x_{0}\right)^{l-i}}{(l-i) !}\right\} d s, \quad l=0,1, \ldots, m-1 .
\end{aligned}
$$

The operators $w_{m}, w_{m-1}, \ldots, w_{0}$ are linear and bounded from the space $E_{q}$ of $(m+1)$-tuples $f=\left(f_{m}(x), f_{m-1}, \ldots, f_{0}\right)$ into the spaces $L_{q}(G), \mathbb{R}, \mathbb{R}, \ldots, \mathbb{R}$ respectively. Therefore, the operator $w=\left(w_{m}, w_{m-1}, \ldots, w_{0}\right): E_{q} \rightarrow E_{q}$ represented by $w f=\left(w_{m} f, w_{m-1} f, \ldots, w_{0} f\right)$ is linear and bounded. By (4.3) and Theorem 1, the operator $w$ is an adjoint operator for the operator $V$ when $1 \leq p<\infty$, in other words, $V^{*}=w$. When $p=\infty, w: E_{1} \rightarrow E_{1}$ is bounded; in this case, the operator $w$ is the restriction of the adjoint operator $V^{*}: E_{\infty}^{*} \rightarrow W_{\infty}^{*}$ of $V$ onto $E_{1} \subset E_{\infty}^{*}$.

(2.3) can be reduced to the following equivalent equation

$$
V S h=z,
$$


with an unknown $h=\left(h_{m}, h_{m-1}, \ldots, h_{0}\right) \in E_{p}$ by the transformation $u=S h$ where $S=N^{-1}$. If $u=S h$, then $u^{(m)}(x)=h_{m}(x), u^{(m-1)}\left(x_{0}\right)=h_{m-1}$, $u^{(m-2)}\left(x_{0}\right)=h_{m-2}, \ldots, u\left(x_{0}\right)=h_{0}$. Hence, (4.3) can be rewritten as

$$
\begin{aligned}
f(V S h) & \equiv \int_{x_{0}}^{x_{1}} f_{m}(x)\left(V_{m} S h\right)(x) d x+\sum_{i=0}^{m-1} f_{i}\left(V_{i} S h\right) \\
& =\int_{x_{0}}^{x_{1}}\left(w_{m} f\right)(\xi) h_{m}(\xi) d \xi+\sum_{i=0}^{m-1}\left(w_{i} f\right) h_{i} \\
& \equiv(w f)(h), \quad \forall f \in E_{q}, \forall h \in E_{p}, 1 \leq p \leq \infty .
\end{aligned}
$$

Therefore, one of the operators $V S$ and $w$ becomes an adjoint operator for the other one. Consequently, the equation

$$
w f=\varphi,
$$

with an unknown function $f=\left(f_{m}(x), f_{m-1}, \ldots, f_{1}, f_{0}\right) \in E_{q}$ and a given function $\varphi=\left(\varphi_{m}(x), \varphi_{m-1}, \ldots, \varphi_{1}, \varphi_{0}\right) \in E_{q}$ can be considered as an adjoint equation of (4.5) (or of (2.3)) for all $1 \leq p \leq \infty$. (4.7) can be written in explicit form as the system of equations

$$
\begin{aligned}
& \left(w_{m} f\right)(\xi)=\varphi_{m}(\xi), \quad \xi \in G, \\
& w_{m-1} f=\varphi_{m-1}, \quad \cdots \quad w_{0} f=\varphi_{0} .
\end{aligned}
$$

By the expressions (4.4), the first equation in (4.8) is an integral equation for $f_{m}(\xi)$ and includes $f_{m-1}, f_{m-2}, \ldots, f_{0}$ as parameters; on the other hand, the other equations in (4.8) constitute a system of $m$ algebraic equations for the unknowns $f_{m-1}, f_{m-2}, \ldots, f_{0}$ and they include some integral functionals defined on $f_{m}(\xi)$. In other words, (4.8) is a system of $m+1$ integro-algebraic equations. This system called the adjoint system for (4.5) (or (2.3)) is constructed by using (4.3) which is actually a formula of integration by parts in a nonclassical form. The traditional type of an adjoint problem is defined by the classical Green's formula of integration by parts [25], therefore, has a sense only for some restricted class of problems.

\section{Solvability Conditions for Completely Nonhomogene- ous Problem}

The operator $Q=w-I_{q}$ is considered where $I_{q}$ is the identity operator on $E_{q}$, i.e. $I_{q} f=f$ for all $f \in E_{q}$. This operator can also be defined as $Q=$ $\left(Q_{m}, Q_{m-1}, \ldots, Q_{0}\right)$ with

$$
\begin{aligned}
& \left(Q_{m} f\right)(\xi)=\left(w_{m} f\right)(\xi)-f_{m}(\xi), \quad \xi \in G, \\
& Q_{i} f=w_{i} f-f_{i}, \quad i=0,1, \ldots, m-1 .
\end{aligned}
$$

By the expressions (4.4) and the conditions imposed on $A_{i}$ and $g_{i}$ for $i=$ $0,1, \ldots, m-1, Q_{m}: E_{q} \rightarrow L_{q}(G)$ is a compact operator, and also $Q_{i}: E_{q} \rightarrow \mathbb{R}$ 
for $i=0,1, \ldots, m-1$ are compact operators where $1<p<\infty$. That is, $Q: E_{q} \rightarrow E_{q}$ is a compact operator, and therefore has a compact adjoint operator $Q^{*}: E_{p} \rightarrow E_{p}$. Since $w=Q+I_{q}$ and $V S=Q^{*}+I_{p}$, where $I_{p}=I_{q}^{*}$, (4.5) and (4.7) are canonical Fredholm type equations, and $S$ is a right regularizer of (2.3) [13]. Consequently, we have the following theorem:

Theorem 2. If $1<p<\infty$, then $V u=0$ has either only the trivial solution or a finite number of linearly independent solutions in $W_{p}$ :

(1) If $V u=0$ has only the trivial solution in $W_{p}$, then also $w f=0$ has only the trivial solution in $E_{q}$. Then, the operators $V: W_{p} \rightarrow E_{p}$ and $w: E_{q} \rightarrow E_{q}$ become linear homeomorphisms.

(2) If $V u=0$ has $n$ linearly independent solutions $u_{1}, u_{2}, \ldots, u_{n}$ in $W_{p}$, then $w f=0$ has also $n$ linearly independent solutions

$f^{\star 1 \star}=\left(f_{m}^{\star 1 \star}(x), f_{m-1}^{\star 1 \star}, \ldots, f_{0}^{\star 1 \star}\right), \ldots, f^{\star n \star}=\left(f_{m}^{\star n \star}(x), f_{m-1}^{\star n \star}, \ldots, f_{0}^{\star n \star}\right)$

in $E_{q}$. In this case, (2.3) and (4.7) have solutions $u \in W_{p}$ and $f \in E_{q}$ for given $z \in E_{p}$ and $\varphi \in E_{q}$ if and only if the conditions

$$
\begin{aligned}
& \int_{x_{0}}^{x_{1}} f_{m}^{\star i \star}(\xi) z_{m}(\xi) d \xi+\sum_{j=0}^{m-1} f_{j}^{\star i \star} z_{j}=0, \quad i=1,2, \ldots, n, \\
& \int_{x_{0}}^{x_{1}} \varphi_{m}(\xi) u_{i}^{(m)}(\xi) d \xi+\sum_{j=0}^{m-1} \varphi_{j} u_{i}^{(j)}\left(x_{0}\right)=0, \quad i=1,2, \ldots, n
\end{aligned}
$$

are satisfied, respectively.

\section{Green's Functional and the Special Adjoint System}

Consider the following equation given in the form of a functional identity

$$
(w f)(u)=u(x), \quad \forall u \in W_{p},
$$

where $f=\left(f_{m}(\xi), f_{m-1}, f_{m-2}, \ldots, f_{0}\right) \in E_{q}$ is an unknown $(m+1)$-tuple and $x \in \bar{G}$ is a parameter.

Definition 1. Suppose that $f(x)=\left(f_{m}(\xi, x), f_{m-1}(x), f_{m-2}(x), \ldots, f_{0}(x)\right) \in$ $E_{q}$ is a $(m+1)$-tuple with parameter $x \in \bar{G}$. If $f=f(x)$ is a solution of (6.1) for a given $x \in \bar{G}$, then $f(x)$ is called a Green's functional of $V$ (or of (2.3)).

Since the operator $I_{W_{p}, C}$ of the imbedding of $W_{p}$ into the space $C(\bar{G})$ of continuous functions on $\bar{G}$ is bounded, the linear functional $\theta(x)$ defined by $\theta(x)(u)=u(x)$ is bounded on $W_{p}$ for a given $x \in \bar{G}$. On the other hand, $(w f)(u)=\left(V^{*} f\right)(u)$. Thus, $(6.1)$ can also be written as $[3,6]$

$$
\left(V^{*} f\right)=\theta(x) \text {. }
$$

In other words, (6.1) can be considered as a special case of the adjoint equation $V^{*} f=\psi$ when $\psi=\theta(x)$. 
By substituting $\alpha=x_{0}$ into (3.1) and using (4.3), we can rewrite (6.1) as

$$
\begin{aligned}
\int_{x_{0}}^{x_{1}}\left(w_{m} f\right)(\xi) u^{(m)}(\xi) d \xi & +\sum_{i=0}^{m-1}\left(w_{i} f\right) u^{(i)}\left(x_{0}\right)=\int_{x_{0}}^{x} \frac{(x-\xi)^{m-1}}{(m-1) !} u^{(m)}(\xi) d \xi \\
& +\sum_{i=0}^{m-1} u^{(i)}\left(x_{0}\right) \frac{\left(x-x_{0}\right)^{i}}{i !}, \quad \forall f \in E_{q}, \quad \forall u \in W_{p} .
\end{aligned}
$$

The components $u^{(m)}(\xi) \in L_{p}(G), u^{(m-1)}\left(x_{0}\right) \in \mathbb{R}, \ldots, u\left(x_{0}\right) \in \mathbb{R}$ of the function $u \in W_{p}$ are unrelated. Thus, we can construct the following system

$$
\begin{aligned}
\left(w_{m} f\right)(\xi) & =\frac{(x-\xi)^{m-1}}{(m-1) !} H(x-\xi), \quad \xi \in G, \\
\left(w_{i} f\right) & =\frac{\left(x-x_{0}\right)^{i}}{i !}, \quad i=m-1, m-2, \ldots, 0
\end{aligned}
$$

where $H(x-\xi)$ is a Heaviside function on $\mathbb{R}$.

(6.1) is equivalent to the system (6.3) which is a special case for the adjoint system (4.8) when

$$
\varphi_{m}(\xi)=\frac{(x-\xi)^{m-1}}{(m-1) !} H(x-\xi), \quad \varphi_{m-1}=\frac{\left(x-x_{0}\right)^{m-1}}{(m-1) !}, \quad \ldots, \quad \varphi_{0}=1 .
$$

Therefore, $f(x)$ is a Green's functional if and only if $f(x)$ is a solution of the system (6.3) for an arbitrary $x \in \bar{G}$. For a solution $u \in W_{p}$ of (2.3) and a Green's functional $f(x)$, we can rewrite (4.3) as

$$
\begin{aligned}
\int_{x_{0}}^{x_{1}} f_{m}(\xi, x) z_{m}(\xi) d \xi+\sum_{i=0}^{m-1} f_{i}(x) z_{i}= & \int_{x_{0}}^{x_{1}} \frac{(x-\xi)^{m-1}}{(m-1) !} H(x-\xi) u^{(m)}(\xi) d \xi \\
& +\sum_{i=0}^{m-1} u^{(i)}\left(x_{0}\right) \frac{\left(x-x_{0}\right)^{i}}{i !}
\end{aligned}
$$

Due to the fact that the right hand side of (6.4) is equal to $u(x)$, we can express the following theorem:

Theorem 3. If (2.3) has at least one Green's functional $f(x)$, then any solution $u \in W_{p}$ of (2.3) can be represented by

$$
u(x)=\int_{x_{0}}^{x_{1}} f_{m}(\xi, x) z_{m}(\xi) d \xi+\sum_{i=0}^{m-1} f_{i}(x) z_{i} .
$$

Additionally, $V u=0$ has only the trivial solution.

Since one of the operators $V: W_{p} \rightarrow E_{p}$ and $w: E_{q} \rightarrow E_{q}$ is a homeomorphism, so is the other, and, there exists a unique Green's functional, where $1 \leq p \leq \infty$. Necessary and sufficient conditions for the existence of a Green's functional can be stated in the following theorem for $1<p<\infty$. 
Theorem 4. If there exists a Green's functional, then it is unique. Additionally, a Green's functional exists if and only if $V u=0$ has only the trivial solution.

The proof of this theorem follows from Theorems 2 and 3.

Remark 1. If $V u=0$ has a nontrivial solution, then a Green's functional corresponding to $V u=z$ does not exist due to Theorem 3. In this case, $V u=z$ usually has no solution unless $z$ is of a specific type. For example, $V u=z$ has no solution unless

$$
\int_{x_{0}}^{x_{1}} f_{m}(\xi) z_{m}(\xi) d \xi+\sum_{i=0}^{m-1} f_{i} z_{i}=0
$$

for at least one solution $f=\left(f_{m}(\xi), f_{m-1}, \ldots, f_{0}\right)$ of the homogeneous adjoint system $w f=0$. Therefore, a representation of the existing solution of $V u=z$ can be constructed by a concept of the generalized Green's functional $[5,6]$.

\section{Some Applications and Comparisons}

In this section, we describe some applications of our method for problems involving complicated nonlocal multipoint boundary conditions in order to demonstrate the advantages of the proposed approach.

Example 1. Firstly, we investigate the following problem, which has been considered in [20], and whose special forms have been discussed in $[8,11]$ :

$$
\begin{aligned}
& u^{(m)}(x)=f(x), \quad x \in G=(0,1), \\
& u(0)=\beta u(\alpha), \quad u^{(1)}(0)=\cdots=u^{(m-2)}(0)=0, \quad u(1)=\gamma u(\eta),
\end{aligned}
$$

where $f(x) \in L_{p}(G), \alpha, \eta \in \bar{G}$ and $\beta, \gamma \in \mathbb{R}$. We can rewrite this problem as

$$
\begin{aligned}
& \left(V_{m} u\right)(x) \equiv u^{(m)}(x)=f(x)=z_{m}(x), \quad x \in G=(0,1), \\
& V_{m-1} u \equiv u(1)-\gamma u(\eta)=0=z_{m-1}, \\
& V_{m-2} u \equiv u^{(m-2)}(0)=0=z_{m-2}, \ldots, V_{1} u \equiv u^{(1)}(0)=0=z_{1}, \\
& V_{0} u \equiv u(0)-\beta u(\alpha)=0=z_{0} .
\end{aligned}
$$

Hence, we have

$$
\begin{aligned}
& a_{m-1}^{i}=\frac{1-\gamma \eta^{i}}{i !}, \quad g_{m-1}(\xi)=\frac{(1-\xi)^{m-1}-\gamma(\eta-\xi)^{m-1} H(\eta-\xi)}{(m-1) !} \\
& a_{m-2}^{i}=\left\{\begin{array}{ll}
1, & \text { for } i=m-2, \\
0, & \text { for } i \neq m-2,
\end{array} \quad g_{m-2}(\xi)=0\right. \\
& a_{m-3}^{i}=\left\{\begin{array}{ll}
1, & \text { for } i=m-3, \\
0, & \text { for } i \neq m-3,
\end{array} \quad g_{m-3}(\xi)=0,\right.
\end{aligned}
$$




$$
\begin{aligned}
& a_{1}^{i}=\left\{\begin{array}{ll}
1, & \text { for } i=1, \\
0, & \text { for } i \neq 1,
\end{array} \quad g_{1}(\xi)=0,\right. \\
& a_{0}^{0}=1-\beta, \quad a_{0}^{j}=-\beta \frac{\alpha^{j}}{j !}, \quad g_{0}(\xi)=-\beta \frac{(\alpha-\xi)^{m-1}}{(m-1) !} H(\alpha-\xi),
\end{aligned}
$$

and $A_{i}(x)=0$ for $i=0,1, \ldots, m-1$ and $j=1, \ldots, m-1$, where $H(\eta-\xi)$ and $H(\alpha-\xi)$ are Heaviside functions on $\mathbb{R}$.

Consequently, the special adjoint system (6.3) corresponding to this problem can be constructed in the following form

$$
\begin{aligned}
f_{m}(\xi)+f_{m-1}\left\{\frac{(1-\xi)^{m-1}-\gamma(\eta-\xi)^{m-1} H(\eta-\xi)}{(m-1) !}\right\} & \\
+f_{0}\left\{-\beta \frac{(\alpha-\xi)^{m-1}}{(m-1) !} H(\alpha-\xi)\right\} & =\frac{(x-\xi)^{m-1}}{(m-1) !} H(x-\xi), \\
f_{m-1}\left\{\frac{1-\gamma \eta^{m-1}}{(m-1) !}\right\}+f_{0}\left\{-\beta \frac{\alpha^{m-1}}{(m-1) !}\right\} & =\frac{x^{m-1}}{(m-1) !}, \\
f_{m-1}\left\{\frac{1-\gamma \eta^{m-2}}{(m-2) !}\right\}+f_{m-2}+f_{0}\left\{-\beta \frac{\alpha^{m-2}}{(m-2) !}\right\} & =\frac{x^{m-2}}{(m-2) !}, \\
f_{m-1}\left\{\frac{1-\gamma \eta^{m-3}}{(m-3) !}\right\}+f_{m-3}+f_{0}\left\{-\beta \frac{\alpha^{m-3}}{(m-3) !}\right\} & =\frac{x^{m-3}}{(m-3) !}, \\
f_{m-1}\left\{\frac{1-\gamma \eta^{2}}{2 !}\right\}+f_{2}+f_{0}\left\{-\beta \frac{\alpha^{2}}{2 !}\right\} & =\frac{x^{2}}{2 !}, \\
f_{m-1}(1-\gamma \eta)+f_{1}+f_{0}(-\beta \alpha) & =x, \\
f_{m-1}(1-\gamma)+f_{0}(1-\beta) & =1,
\end{aligned}
$$

where $\xi \in(0,1)$. First, we determine $f_{m-1}$ and $f_{0}$ by using only (7.3) and (7.8) under the condition $\Delta=(1-\beta)\left(1-\gamma \eta^{m-1}\right)+(1-\gamma) \beta \alpha^{m-1} \neq 0$ in order to solve (7.3)-(7.8), and then the others. Thus, we have

$$
\begin{aligned}
& f_{m-1}=\frac{1}{\Delta}\left\{(1-\beta) x^{m-1}+\beta \alpha^{m-1}\right\}, \\
& f_{i}=\frac{x^{i}}{i !}+f_{0} \beta \frac{\alpha^{i}}{i !}-f_{m-1}\left\{\frac{1-\gamma \eta^{i}}{i !}\right\} \text { for } i=1,2, \ldots, m-2, \\
& f_{0}=\frac{1}{\Delta}\left\{1-\gamma \eta^{m-1}-(1-\gamma) x^{m-1}\right\} .
\end{aligned}
$$

After substituting $f_{m-1}$ and $f_{0}$ into the first equation from which $f_{m}(\xi)$ will be obtained, $f_{m}(\xi)$ can be written as

$$
\begin{aligned}
f_{m}(\xi)= & \frac{(x-\xi)^{m-1}}{(m-1) !} H(x-\xi) \\
& +\frac{1}{\Delta}\left\{1-\gamma \eta^{m-1}-(1-\gamma) x^{m-1}\right\}\left\{\beta \frac{(\alpha-\xi)^{m-1}}{(m-1) !} H(\alpha-\xi)\right\} \\
& -\frac{1}{\Delta}\left\{(1-\beta) x^{m-1}+\beta \alpha^{m-1}\right\}\left\{\frac{(1-\xi)^{m-1}-\gamma(\eta-\xi)^{m-1} H(\eta-\xi)}{(m-1) !}\right\} .
\end{aligned}
$$


Thus, Green's functional $f(x)=\left(f_{m}(\xi, x), f_{m-1}(x), f_{m-2}(x), \ldots, f_{0}(x)\right)$ for the problem has been determined. The first component $f_{m}(\xi, x)=f_{m}(\xi)$ is Green's function for the problem. After substituting $\xi=s$ for notational compatibility, it can be seen easily that $f_{m}(\xi, x)$ is equal to Green's function constructed in [20] for the problem. By (6.5) in Theorem 3, the representative solution to the problem can be given as

$$
\begin{aligned}
u(x)= & \int_{0}^{1}\left[\frac{(x-\xi)^{m-1}}{(m-1) !} H(x-\xi)+\frac{1}{\Delta}\left\{1-\gamma \eta^{m-1}-(1-\gamma) x^{m-1}\right\}\right. \\
& \times\left\{\beta \frac{(\alpha-\xi)^{m-1}}{(m-1) !} H(\alpha-\xi)\right\}-\frac{1}{\Delta}\left\{(1-\beta) x^{m-1}+\beta \alpha^{m-1}\right\} \\
& \left.\times\left\{\frac{(1-\xi)^{m-1}-\gamma(\eta-\xi)^{m-1} H(\eta-\xi)}{(m-1) !}\right\}\right] f(\xi) d \xi
\end{aligned}
$$

Example 2. Now, we consider the following problem whose special form has been studied in [21]:

$$
\begin{aligned}
u^{(m)}(x) & =f(x), \quad x \in G=(0,1), \\
u(1) & =\gamma_{1} \int_{0}^{1} u(t) d t, \\
u^{(k)}(0) & =0 \quad \text { for } k=1,2, \ldots, m-2, \\
u(0) & =\gamma_{0} \int_{0}^{1}(1+t) u(t) d t,
\end{aligned}
$$

where $f(x) \in L_{p}(G)$ and $\gamma_{0}, \gamma_{1} \in \mathbb{R}$. We can rewrite this problem as

$$
\begin{aligned}
& \left(V_{m} u\right)(x) \equiv u^{(m)}(x)=f(x)=z_{m}(x), \quad x \in G=(0,1) \\
& V_{m-1} u \equiv u(1)-\gamma_{1} \int_{0}^{1} u(t) d t=0=z_{m-1} \\
& V_{k} u \equiv u^{(k)}(0)=0=z_{k} \text { for } k=1,2, \ldots, m-2 \\
& V_{0} u \equiv u(0)-\gamma_{0} \int_{0}^{1}(1+t) u(t) d t=0=z_{0}
\end{aligned}
$$

Hence, we have $A_{i}(x)=0$ and

$$
\begin{aligned}
& a_{m-1}^{i}=\frac{1}{i !}\left(1-\frac{\gamma_{1}}{i+1}\right), \quad g_{m-1}(\xi)=\frac{(1-\xi)^{m-1}}{(m-1) !}\left\{1-\frac{\gamma_{1}(1-\xi)}{m}\right\} \\
& a_{r}^{i}=\left\{\begin{array}{l}
1, \quad \text { for } i=r, \\
0, \quad \text { for } i \neq r, \quad g_{r}(\xi)=0 \quad \text { for } r=1,2, \ldots, m-2,
\end{array}\right. \\
& a_{0}^{0}=1-\frac{3 \gamma_{0}}{2}, \quad a_{0}^{j}=-\frac{\gamma_{0}(2 j+3)}{(j+2) !}, \quad g_{0}(\xi)=-\frac{\gamma_{0}(1-\xi)^{m}(1+2 m+\xi)}{(m+1) !},
\end{aligned}
$$

for $i=0,1, \ldots, m-1$ and $j=1, \ldots, m-1$. In this case, the special adjoint 
system (6.3) corresponding to this problem becomes in the following form

$$
\begin{aligned}
& f_{m}(\xi)+f_{m-1} \frac{(1-\xi)^{m-1}}{(m-1) !}\left\{1-\frac{\gamma_{1}(1-\xi)}{m}\right\} \\
& \quad-f_{0} \frac{\gamma_{0}(1-\xi)^{m}(1+2 m+\xi)}{(m+1) !}=\frac{(x-\xi)^{m-1}}{(m-1) !} H(x-\xi), \\
& f_{m-1} \frac{1}{(m-1) !}\left(1-\frac{\gamma_{1}}{m}\right)-f_{0} \frac{\gamma_{0}(2 m+1)}{(m-1) ! m(m+1)}=\frac{x^{m-1}}{(m-1) !}, \\
& f_{m-1} \frac{1}{k !}\left(1-\frac{\gamma_{1}}{k+1}\right)+f_{k}-f_{0} \frac{\gamma_{0}(2 k+3)}{k !(k+1)(k+2)}=\frac{x^{k}}{k !} \text { for } k=1,2, \ldots, m-2, \\
& f_{m-1}\left(1-\gamma_{1}\right)+f_{0}\left(1-\frac{3 \gamma_{0}}{2}\right)=1,
\end{aligned}
$$

where $\xi \in(0,1)$. Similarly, we firstly find the unknowns $f_{m-1}$ and $f_{0}$ by using only (7.13) and (7.14) under the condition

$$
\Delta=\left(1-\frac{\gamma_{1}}{m}\right)\left(1-\frac{3 \gamma_{0}}{2}\right)+\frac{\left(1-\gamma_{1}\right) \gamma_{0}(2 m+1)}{m(m+1)} \neq 0
$$

in order to solve (7.13)-(7.14), and then the others. Thus, we obtain

$$
\begin{aligned}
& f_{m-1}=\frac{1}{\Delta}\left\{\left(1-\frac{3 \gamma_{0}}{2}\right) x^{m-1}+\frac{\gamma_{0}(2 m+1)}{m(m+1)}\right\} \\
& f_{k}=\frac{x^{k}}{k !}+\frac{f_{0} \gamma_{0}(2 k+3)}{k !(k+1)(k+2)}-\frac{f_{m-1}}{k !}\left(1-\frac{\gamma_{1}}{k+1}\right) \text { for } k=1,2, \ldots, m-2, \\
& f_{0}=\frac{1}{\Delta}\left\{1-\frac{\gamma_{1}}{m}-\left(1-\gamma_{1}\right) x^{m-1}\right\} .
\end{aligned}
$$

After substituting $f_{m-1}$ and $f_{0}$ into the first equation from which $f_{m}(\xi)$ will be identified, $f_{m}(\xi)$ can be derived as

$$
\begin{aligned}
f_{m}(\xi)= & \frac{(x-\xi)^{m-1}}{(m-1) !} H(x-\xi) \\
& +\frac{1}{\Delta}\left\{1-\frac{\gamma_{1}}{m}-\left(1-\gamma_{1}\right) x^{m-1}\right\} \frac{\gamma_{0}(1-\xi)^{m}(1+2 m+\xi)}{(m+1) !} \\
& -\frac{1}{\Delta}\left\{\left(1-\frac{3 \gamma_{0}}{2}\right) x^{m-1}+\frac{\gamma_{0}(2 m+1)}{m(m+1)}\right\} \frac{(1-\xi)^{m-1}}{(m-1) !}\left\{1-\frac{\gamma_{1}(1-\xi)}{m}\right\} .
\end{aligned}
$$

As a result, Green's functional $f(x)=\left(f_{m}(\xi, x), f_{m-1}(x), f_{m-2}(x), \ldots, f_{0}(x)\right)$ for the problem has been determined. The first component $f_{m}(\xi, x)=f_{m}(\xi)$ is Green's function for the problem. After substituting $\xi=s$ and $m=2$ into the statement $f_{m}(\xi, x)$ for notational compatibility, it can be seen easily that $f_{m}(\xi, x)$ is the negative one of Green's function constructed in [21] for the special case $m=2$ of the problem. Because we consider the operator $V_{m} u \equiv u^{(m)}$ instead of $V_{m} u \equiv-u^{(m)}$. By (6.5) in Theorem 3, the representative 
solution to the general problem can be given as

$$
\begin{aligned}
u(x) & =\int_{0}^{1}\left[\frac{(x-\xi)^{m-1}}{(m-1) !} H(x-\xi)+\frac{1}{\Delta}\left\{1-\frac{\gamma_{1}}{m}-\left(1-\gamma_{1}\right) x^{m-1}\right\}\right. \\
& \times \frac{\gamma_{0}(1-\xi)^{m}(1+2 m+\xi)}{(m+1) !}-\frac{1}{\Delta}\left\{\left(1-\frac{3 \gamma_{0}}{2}\right) x^{m-1}+\frac{\gamma_{0}(2 m+1)}{m(m+1)}\right\} \\
& \left.\times \frac{(1-\xi)^{m-1}}{(m-1) !}\left\{1-\frac{\gamma_{1}(1-\xi)}{m}\right\}\right] f(\xi) d \xi .
\end{aligned}
$$

Example 3. Finally, we consider a nonlinear problem for which and whose special form Green's functions have been presented in $[7,19]$ respectively:

$$
\begin{aligned}
& u^{(m)}(x)=-a(x) f(u(x)), \quad x \in G=(0,1), \\
& u(1)=\sum_{i=1}^{n-2} \alpha_{i} u\left(\eta_{i}\right), \quad u^{(k)}(0)=0 \quad \text { for } k=0,1, \ldots, m-2,
\end{aligned}
$$

where $\alpha_{i}>0$ for $i=1,2, \ldots, n-2$ are real numbers, $a(x): G \rightarrow[0, \infty)$ is a continuous function $(\in C(G)), f(u(x)):[0, \infty) \rightarrow[0, \infty)$ is a continuous function and $0<\eta_{1}<\eta_{2}<\cdots<\eta_{n-2}<1$. We can rewrite this problem by our notation as

$$
\begin{aligned}
& \left(V_{m} u\right)(x) \equiv u^{(m)}(x)=-a(x) f(u(x))=z_{m}(x), \quad x \in G=(0,1), \\
& V_{m-1} u \equiv u(1)-\sum_{i=1}^{n-2} \alpha_{i} u\left(\eta_{i}\right)=0=z_{m-1}, \\
& V_{k} u \equiv u^{(k)}(0)=0=z_{k} \quad \text { for } k=0,1, \ldots, m-2 .
\end{aligned}
$$

Hence, we have $A_{r}(x)=0$ and $a_{m-1}^{r}=\left(1-\sum_{i=1}^{n-2} \alpha_{i} \eta_{i}^{r}\right) / r !$,

$$
\begin{aligned}
& g_{m-1}(\xi)=\frac{1}{(m-1) !}\left[(1-\xi)^{m-1}-\sum_{i=1}^{n-2} \alpha_{i}\left(\eta_{i}-\xi\right)^{m-1} H\left(\eta_{i}-\xi\right)\right], \\
& a_{k}^{r}=\left\{\begin{array}{ll}
1, & \text { for } r=k, \\
0, & \text { for } r \neq k,
\end{array} g_{k}(\xi)=0 \quad \text { for } k=0,1, \ldots, m-2,\right.
\end{aligned}
$$

for $r=0,1, \ldots, m-1$. In this case, the special adjoint system (6.3) corresponding to this problem becomes in the following form

$$
\begin{aligned}
& f_{m}(\xi)+f_{m-1} \frac{1}{(m-1) !}\left[(1-\xi)^{m-1}\right. \\
& \left.\quad-\sum_{i=1}^{n-2} \alpha_{i}\left(\eta_{i}-\xi\right)^{m-1} H\left(\eta_{i}-\xi\right)\right]=\frac{(x-\xi)^{m-1}}{(m-1) !} H(x-\xi), \\
& \frac{f_{m-1}}{(m-1) !}\left(1-\sum_{i=1}^{n-2} \alpha_{i} \eta_{i}^{m-1}\right)=\frac{x^{m-1}}{(m-1) !}, \\
& \frac{f_{m-1}}{k !}\left(1-\sum_{i=1}^{n-2} \alpha_{i} \eta_{i}^{k}\right)+f_{k}=\frac{x^{k}}{k !} \quad \text { for } k=0,1, \ldots, m-2,
\end{aligned}
$$


where $\xi \in(0,1)$. Similarly, we firstly determine the unknown $f_{m-1}$ from (7.19) under the condition $1-\sum_{i=1}^{n-2} \alpha_{i} \eta_{i}^{m-1} \neq 0$ in order to solve (7.19)-(7.20), and then the others. Thus, we obtain

$$
f_{m-1}=\frac{x^{m-1}}{1-D}, \quad f_{k}=\frac{x^{k}}{k !}-\frac{x^{m-1}}{(1-D) k !}\left(1-\sum_{i=1}^{n-2} \alpha_{i} \eta_{i}^{k}\right) \text { for } k=0,1, \ldots, m-2
$$

where $D=\sum_{i=1}^{n-2} \alpha_{i} \eta_{i}^{m-1}$. After substituting $f_{m-1}$ into the first equation from which $f_{m}(\xi)$ will be identified, $f_{m}(\xi)$ can be derived as

$$
\begin{aligned}
f_{m}(\xi)= & \frac{(x-\xi)^{m-1}}{(m-1) !} H(x-\xi) \\
& -\frac{x^{m-1}}{(1-D)(m-1) !}\left[(1-\xi)^{m-1}-\sum_{i=1}^{n-2} \alpha_{i}\left(\eta_{i}-\xi\right)^{m-1} H\left(\eta_{i}-\xi\right)\right] .
\end{aligned}
$$

Consequently, Green's functional $f(x)=\left(f_{m}(\xi, x), f_{m-1}(x), f_{m-2}(x), \ldots\right.$, $\left.f_{0}(x)\right)$ for the problem has been determined. Its first component $f_{m}(\xi, x)=$ $f_{m}(\xi)$ is Green's function for the problem. After substituting $\xi=s$ into the statement $f_{m}(\xi, x)$ for notational compatibility, it can be seen easily that $f_{m}(\xi, x)$ is equal to Green's function constructed in [19]. By (6.5) in Theorem 3, we have

$$
\begin{aligned}
u(x)= & -\int_{0}^{1}\left\{\frac{(x-\xi)^{m-1}}{(m-1) !} H(x-\xi)-\frac{x^{m-1}}{(1-D)(m-1) !}\left[(1-\xi)^{m-1}\right.\right. \\
& \left.\left.-\sum_{i=1}^{n-2} \alpha_{i}\left(\eta_{i}-\xi\right)^{m-1} H\left(\eta_{i}-\xi\right)\right]\right\} a(\xi) f(u(\xi)) d \xi
\end{aligned}
$$

for the representative solution to the problem. As can be noticed, (7.21) is a nonlinear integral equation for $u(x)$. In other words, a solution $u(x) \in W_{p}$ to the problem is equivalent to the solution of integral equation $(7.21)$ in $C(G)$.

Henceforth the solution to this integral equation can be investigated under the considered assumptions by using the following iteration

$$
u_{N+1}(x)=\left(\mathcal{A} u_{N}\right)(x) \text { for } N=0,1, \ldots
$$

where

$$
\begin{array}{r}
\left(\mathcal{A} u_{N}\right)(x)=-\int_{0}^{1}\left\{\frac{(x-\xi)^{m-1}}{(m-1) !} H(x-\xi)-\frac{x^{m-1}}{(1-D)(m-1) !}\left[(1-\xi)^{m-1}\right.\right. \\
\left.\left.-\sum_{i=1}^{n-2} \alpha_{i}\left(\eta_{i}-\xi\right)^{m-1} H\left(\eta_{i}-\xi\right)\right]\right\} a(\xi) f\left(u_{N}(\xi)\right) d \xi
\end{array}
$$

and $u_{0}(x)$ is an appropriate initial approximation. If $\mathcal{A}$ is a contraction operator, then $u(x)=\lim _{N \rightarrow \infty} u_{N}(x)$ by Banach fixed point theorem. 


\section{Conclusions}

As can be seen from the theoretical presentation and illustrative applications, the proposed approach principally differs from the known classical methods used for the construction of Green's function, it is based on the usage of the structural properties of the space of solutions instead of the classical Green's formula of integration by parts, it minimizes the difficulties emphasized in the introduction, and it has a natural and constructive property which can be easily applied to a very wide class of linear and some nonlinear boundary value problems involving linear nonlocal nonclassical multipoint conditions with also integral-type terms. Because of these properties, it is one of the scarce methods which are focused on the derivation of a solution to such problems by reducing to an integral equation in general.

Green's function for the $m$-order problem in the focus of the work is a special form of Green's functional for same problem. Moreover, the introduced special adjoint system corresponding to that problem allows us to get an idea about the existence and uniqueness of the solutions for the problem such that a unique solution to the special adjoint system exists if and only if Green's function uniquely exists subject to the solvability conditions of the problem.

Finally, the proposed approach can successfully be employed also for the problems resulting from the addition of some delayed or loaded (forced) terms to the operator $V_{m}$ as long as the linearity for the operator is conserved.

\section{Acknowledgements}

The authors are thankful to the anonymous reviewers for their suggestions and comments which improved the present paper considerably.

\section{References}

[1] S.S. Akhiev. Representations of the solutions of some linear operator equations. Soviet Math. Dokl., 21(2):555-558, 1980.

[2] S.S. Akhiev. Fundamental solutions of functional differential equations and their representations. Soviet Math. Dokl., 29(2):180-184, 1984.

[3] S.S. Akhiev. A new fundamental solution concept and application to some local and nonlocal problems. Bull. Tech. Univ. Istanbul, 47(3):93-99, 1994.

[4] S.S. Akhiev. Solvability conditions and Green functional concept for local and nonlocal linear problems for a second order ordinary differential equation. Math. Comput. Appl., 9(3):349-358, 2004.

[5] S.S. Akhiev. Green and generalized Green's functionals of linear local and nonlocal problems for ordinary integro-differential equations. Acta Appl. Math., 95:73-93, 2007. http://dx.doi.org/10.1007/s10440-006-9056-z.

[6] S.S. Akhiev and K. Oruçoğlu. Fundamental solutions of some linear operator equations and applications. Acta Appl. Math., 71:1-30, 2002. http://dx.doi.org/10.1023/A:1014529217268.

[7] P.W. Eloe and B. Ahmad. Positive solutions of a nonlinear $n$th order boundary value problem with nonlocal conditions. Appl. Math. Lett., 18(5):521-527, 2005. http://dx.doi.org/10.1016/j.aml.2004.05.009. 
[8] X. Hao, L. Liu and Y. Wu. Positive solutions for nonlinear $n$ th-order singular nonlocal boundary value problems. Bound. Value Probl., pp. 1-10, 2007. http://dx.doi.org/10.1155/2007/74517.

[9] J. Henderson. Existence and uniqueness of solutions of $(k+2)$-point nonlocal boundary value problems for ordinary differential equations. Nonlinear Anal., 74(7):2576-2584, 2011. http://dx.doi.org/10.1016/j.na.2010.11.048.

[10] J. Henderson and C.J. Kunkel. Uniqueness of solutions of linear nonlocal boundary value problems. Appl. Math. Lett., 21:1053-1056, 2008.

http://dx.doi.org/10.1016/j.aml.2006.06.024.

[11] Y. Ji and Y. Guo. The existence of countably many positive solutions for nonlinear $n$ th-order three-point boundary value problems. Bound. Value Probl., pp. 1-18, 2009. http://dx.doi.org/10.1155/2009/572512.

[12] L.V. Kantorovich and G.P. Akilov. Functional analysis. Pergamon Press, New York, 1982.

[13] S.G. Krein. Linear Equations in Banach Space. Nauka, Moscow, Russia, 1971. (in Russian)

[14] C. Lanczos. Linear Differential Operators. D. Van Nostrand Company Ltd., 1964.

[15] Y. Liu and D. O'Regan. Multiplicity results using bifurcation techniques for a class of fourth-order m-point boundary value problems. Bound. Value Probl., pp. 1-20, 2009. http://dx.doi.org/10.1155/2009/970135.

[16] M.A. Naimark. Linear Differential Operators. Nauka, Moscow, Russia, 1969. (in Russian)

[17] K. Orucoglu. A new Green function concept for fourth-order differential equations. Electron. J. Differential Equations, 28:1-12, 2005.

[18] K. Ozen and K. Orucoglu. Investigation of numerical solution for fourth-order nonlocal problem by the reproducing kernel method. In T.E. Simos, G. Psihoyios, C. Tsitouras and Z. Anastassi(Eds.), Numerical Analysis And Applied Mathematics (ICNAAM 2011), volume 1389 of AIP Conf. Proc., pp. 1164-1167, Halkidiki, Greece, 2011. American Institute of Physics. http://dx.doi.org/10.1063/1.3637822.

[19] C. Pang, W. Dong and Z. Wei. Green's function and positive solutions of $n$th order $m$-point boundary value problem. Appl. Math. Comput., 182(2):12311239, 2006. http://dx.doi.org/10.1016/j.amc.2006.05.10.

[20] S. Roman. Linear differential equation with additional conditions and formulae for Green's function. Math. Model. Anal., 16(3):401-417, 2011. http://dx.doi.org/10.3846/13926292.2011.602125.

[21] S. Roman and A. Štikonas. Green's functions for stationary problems with nonlocal boundary conditions. Lith. Math. J., 49(2):190-202, 2009. http://dx.doi.org/10.1007/s10986-009-9041-0.

[22] S. Roman and A. Štikonas. Third-order linear differential equation with three additional conditions and formula for Green's function. Lith. Math. J., 50(4):426446, 2010. http://dx.doi.org/10.1007/s10986-010-9097-x.

[23] S. Roman and A. Štikonas. Green's function for discrete second-order problems with nonlocal boundary conditions. Bound. Value Probl., 2011:1-23, 2011. http://dx.doi.org/10.1155/2011/767024. Article ID 767024 
[24] G.E. Shilov. Generalized Functions and Partial Differential Equations. Gordon and Breach, New York, 1968.

[25] I. Stakgold. Green's Functions and Boundary Value Problems. WileyInterscience Publications, New York, 1998.

[26] A. Štikonas. Investigation of characteristic curve for Sturm-Liouville problem with nonlocal boundary conditions on torus. Math. Model. Anal., 16(1):1-22, 2011. http://dx.doi.org/10.3846/13926292.2011.552260.

[27] A. Štikonas and S. Roman. Stationary problems with two additional conditions and formulae for Green's function. Numer. Funct. Anal. Optim., 30(9-10):11251144, 2009. http://dx.doi.org/10.1080/01630560903420932.

[28] S. Timoshenko. Theory of Elastic Stability. McGraw-Hill, New York, 1961.

[29] V.S. Vladimirov. Equations of Mathematical Physics. Marcel Dekker, New York, 1971. 